\title{
Integrated hardware/software in the Algeria Telecom access layers NGN model:MA5600T and C300M Shelfs MSAN solutions
}

\author{
Samir Ghouali, ${ }^{* 1,2}$ Mohammed Feham, ${ }^{2}$ and Rachid Merzougui ${ }^{2}$ \\ ${ }^{1}$ Faculty of Sciences and Technology, Mustapha Stambouli University, 29000 Mascara, Algeria \\ ${ }^{2}$ Faculty of Engineering Science of Tlemcen, STIC Laboratory, Tlemcen, Algeria
}

Received June 04, 2019; accepted June 17, 2019; published June 30, 2019

\begin{abstract}
The evolution of an existing network to the new structure will require a phased migration strategy aimed at minimising capital expenditure during the transition phase, while reaping the benefits early on. Any action taken during this transition step should simplify the network's evolution to the NGN packet-switched architecture. This paper presents the development of PSTN networks into NGN Optical Fiber, its configuration and implementation of Smart AX MA5600T HUAWEI and C300M ZTE Shelfs MSANs solutions.
\end{abstract}

The fixed access network plays an increasingly important role in terms of investment, revenue and number of subscribers in the telecommunications systems landscape. The growing demand for bandwidth-intensive services has attracted the attention of operators by encouraging them to review their network architecture and migrate to an IPbased Next Generation Network (NGN) model for the transport of all types of traffic [1-2].

Since it was impossible to create this network overnight, Algeria Telecom followed the best scenario in order to create it in accordance with standards, starting with the upper layers (service management, control, transport) with the implementation of Soft switch and Medias Gateway, then moving down to the transport layer, with the implementation of the routers that constitute the IP/MPLS backbone. Thus, the implementation of the RMS (Multiservice Network) has made it possible to ensure easy integration of existing networks and gives the possibility of offering new services to customers.

In the context of this migration, the implementation of MSAN Multi-Service Access Node (MSAN cabinet consists of narrowband (POTS), broadband (xDSL) services, batteries with rectifiers, optical transmission unit and copper distribution) at an access network level is the optimal solution to meet the demand for services and to guarantee the existing services with a better signal quality. Multi-Service Access Node (MSAN) is a type of FTTN network, where fibers are connected to an existing node which is serving users with a copper pair but below the required bandwidth $[1,4]$.

\footnotetext{
*E-mail: s.ghouali@univ-mascara.dz
}

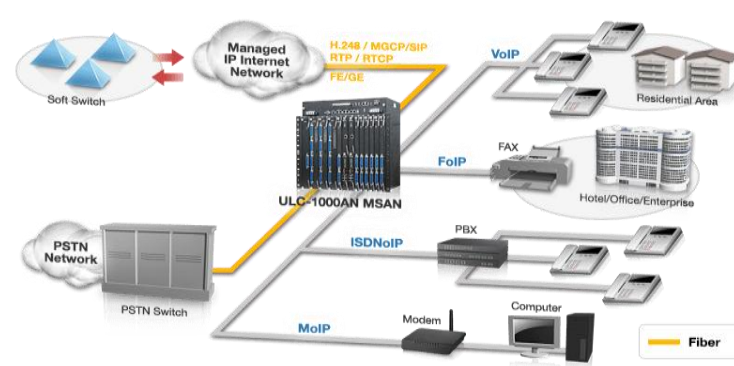

Fig. 1. Multi-Service Access Node Solution [2].

This article is a practical study that presents the commissioning and configuration of the two MSANs installed in Algeria Smart AX MA5600T and C300M.

Algeria Telecom has decided to improve and develop its fixed network through the implementation of the MSAN solution. It enables the transition between the RTC and the NGN, the aim of which is to offer more reliable services to the end user.

MSAN is the access node in NGN networks, it can provide multiple voice and data services simultaneously with high capacity and reliability [3,5-6].

Before the deployment of MSAN in fixed networks, there was a separation between voice and data flow, MSAN is a powerful solution that replaces DSLAM for data services and AXIS for voice services. It includes POTS, Ethernet, xDSL and FTTx cards to provide converged technology over IP, among these features [4] are:

-The coding and media packaging stream received from the access network to the packet network and vice versa, in other words, the conversion of traffic, for example TDM/IP,

-Media Transmission flows according to the instructions of the Media Gateway Controller,

-Supports H.248, MGCP and SIP protocols,

-Supports real time transport protocol RTP/RTCP,

-Supports G.711, G.729, G.723.1 audio codecs,

-Supports high speed interfaces: ADSL/ADSL2+, VDSL2, SHDSL, GPON and Ethernet,

-Supports PSTN, ISDN, FAX, V5 interface, xDSL and GPON services. 
The practical part focuses on different steps of commissioning and configuration of the Smart AX MA5600T and C300M Shelfs MSAN. After hardware installation and distribution cabling of an MSAN indoor site, the next step is designed for the commissioning and configuration of broadband and narrowband services. There are two configuration modes [3, 5-6, 9]:

- Configuration via the console port: The console port located in the MSAN control board is used when commissioning or configuring the MSAN locally.

-Telnet configuration (via Ethernet port): In this mode, an Ethernet cable is used to configure the MSAN connected to the Eth port of the control board, the Ethernet cable is used to configure or manage the equipment locally and remotely. The maintenance terminal IP address must be configured and in the same subnetwork as the MSAN MEth address.

After ensuring the connection between the terminal and our MSAN, it is necessary to use a terminal emulator on which the configuration commands are entered, there are several emulators for this function (e.g. TERATERM) just configure the MSAN Eth IP address, port and access mode (Telnet or SSH).

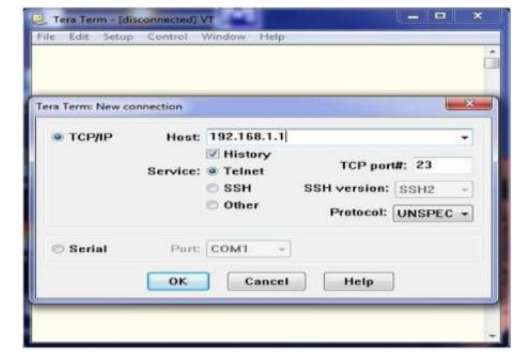

Fig. 2. Telnet Configuration Via TERATERM.

In this section, we will show how to configure the chassis and the different boards of Smart AX MA5600T and C300M [5, 9].

The configuration of the cards is done in two possible ways: Auto-configuration - this configuration method is generally used during commissioning, using the following commands (Table 1), the type of cards inserted will be detected automatically;

Table 1. Auto cards configuration

\begin{tabular}{|l|l|}
\hline MSAN Smart AX MA5600T & MSAN C300M \\
\hline huawei(config)\# boardconfirm 0 & ZXAN(config)\#set-pnpenable \\
\hline
\end{tabular}

Manual configuration, where to add a card to a slot, you can use the following commands (Table 2):

Table 2. Manual cards configuration

\begin{tabular}{|l|ll|}
\hline MSAN Smart AX MA5600T & MSAN C300M \\
\hline huawei(config)\#board add 0/2 & \multicolumn{2}{l|}{ ZXAN(config)\#add-card rack no } \\
h802gpbd & $1 \quad$ shelfno $1 \quad$ slotno \\
0 frame 2 slot board added & VSWEC & \\
successfully & ZXAN(config)\#exit \\
huawei(config)\#display board & & \\
$0 / 2$ & & \\
\hline
\end{tabular}

Board Name:H802GPBD

BoardStatus: Failed

VLAN's declaration, assignment and Management: In this part, we will show how to declare a VLAN and how to assign it to a port (Table 3).

Table 3. VLAN's Declaration, Assignment and Management

\begin{tabular}{|c|c|}
\hline MSAN Smart AX MA5600T & MSAN C300M \\
\hline huawei (config)\#vlan 50 smart & $\begin{array}{l}\text { ZXAN(config)\#vlan } 1000 \\
\text { ZXAN(config-vlan)\#exit }\end{array}$ \\
\hline $\begin{array}{l}\text { huawei(config)\#port vlan } 200 / 9 \\
0 \\
\text { huawei(config)\#port vlan } 210 / 9 \\
0\end{array}$ & $\begin{array}{l}\text { ZXAN(config)\#interface } \\
\text { gei_1/21/1 } \\
\text { ZXAN(config-if)\#switchport } \\
\text { mode hybrid } \\
\text { ZXAN(config-if)\# switch port } \\
\text { vlan } 20 \text { tag } \\
\text { ZXAN(config-if)\# switch port } \\
\text { vlan } 21 \text { tag } \\
\text { ZXAN (config-if)\#exit }\end{array}$ \\
\hline $\begin{array}{l}\text { huawei(config)\#vlan } \\
\text { standard } \\
\text { huawei(config)\#port vlan } 10 \\
0 / 101 \\
\text { huawei(config)\#interface vlanif } \\
10 \\
\text { huawei(config-if-vlanif 10) \# ip } \\
\text { address } 172.28 .1 .43 \\
\text { 255.255.255.0 description } \\
\text { MSAN-Management }\end{array}$ & $\begin{array}{l}\text { ZXAN(config)\#vlan } \\
\text { ZXAN(config-vlan)\#description } \\
\text { MNG } \\
\text { ZXAN(config)\#interface mng1 } \\
\text { ZXAN(config-if)\#ip address } \\
136.1 .1 .100 \text { 255.255.0.0 }\end{array}$ \\
\hline
\end{tabular}

SNMP protocol configuration: In this step we will quote the essential commands to configure the SNMP management protocol [4]:

1. Define the initial parameters, namely, the version and the authorization to read or modify the data (Table 4).

Table 4. Define the initial SNMP parameters

\begin{tabular}{|l|l|}
\hline MSAN Smart AX MA5600T & MSAN C300M \\
\hline $\begin{array}{l}\text { huawei(config)\#snmp-agent } \\
\text { community read name1 } \\
\text { huawei(config)\#snmp-agent } \\
\begin{array}{l}\text { community write name2 } \\
\text { huawei(config)\#snmp-agent sys- } \\
\text { info version v2c }\end{array}\end{array}$ & $\begin{array}{l}\text { Community private view all } \\
\text { view rw }\end{array}$ \\
\hline
\end{tabular}

2. Identify the target host address that is configured to receive the sent messages (Table 5).

Table 5. Target Host Address

\begin{tabular}{|l|l|l|}
\hline \multicolumn{2}{|l|}{ MSAN Smart AX MA5600T } & \multicolumn{2}{l|}{ MSAN C300M } \\
\hline huawei(config)\#snmp-agent & \multicolumn{2}{|l|}{ ZXAN(config)\#snmp-server } \\
target-host trap-hostname & host 192.168.103.20 trap \\
U2000_Activerraderess & version 2c public enable \\
192.168.103.20 trap-params & notifications isnms server \\
name u2000 & & server-index 1 udp-port 162 \\
\hline
\end{tabular}

3. Packets Monitor sent to the NMS server so that they are checked when the alarm is reported (Table 6).

Table 6. Package Supervision

\begin{tabular}{|l|l|}
\hline MSAN Smart AX MA5600T & MSAN C300M \\
\hline $\begin{array}{l}\text { huawei(config)\#snmp-agent trap } \\
\text { enable standard }\end{array}$ & $\begin{array}{l}\text { ZXAN(config)\#snmp-server } \\
\text { enabletrap SNMP }\end{array}$ \\
\hline
\end{tabular}


ADSL2+ service configuration: in this section we propose to configure the ADSL 2+ service in both Smart AX MA5600T and C300M, for this, we will mention the main steps of this configuration:

1. Creating VLANs: this step consists in creating a smart

VLAN for the ADSL 2+ services (Table 7).

Table 7. VLAN ADSL +2 services

\begin{tabular}{|l|l|}
\hline MSAN Smart AX MA5600T & MSAN C300M \\
\hline huawei(config)\#vlan 50 smart & ZXAN (config)\#vlan 1000 \\
& ZXAN (config-vlan)\#exit \\
\hline
\end{tabular}

2. Uplink ports Configurations: in this step, we will configure the ports of the GE interfaces and then associate a VLAN to each port (Table 8).

Table 8. Uplink Ports Configurations

\begin{tabular}{|c|c|}
\hline MSAN Smart AX MA5600T & MSAN C300M \\
\hline $\begin{array}{l}\text { huawei(config)\#port vlan } 50 \\
0 / 190\end{array}$ & $\begin{array}{l}\text { ZXAN(config)\#interface } \\
\text { gei } 1 / 21 / 1\end{array}$ \\
\hline $\begin{array}{l}\text { huawei(config)\#port vlan } 50 \\
0 / 191\end{array}$ & $\begin{array}{l}\text { ZXAN(config-if)\#switchport } \\
\text { mode hybrid }\end{array}$ \\
\hline huawei(config)\#link-aggregation & ZXAN(config-if)\#switchport \\
\hline $\begin{array}{lllll}0 / 19 & 0 & 0 / 19 & 1 & \text { egress ingress }\end{array}$ & vlan $1000 \mathrm{tag}$ \\
\hline work model acp-static & ZXAN(config-if)\#exit \\
\hline
\end{tabular}

3. ADSL 2 + models' creation: In most cases these models are configured by default, which neglects the passage through this step.

A template is the combination of a "channel-profile" that specifies the subscriber's flow rate and a "line-profile". The profile of a subscriber line is defined by a template that describes the different parameters of this line (ADSL, VDSL, Data Rate, Transmission Type, Signal-To-Noise ratio, etc.).

It should be noted that when adding a model, the model index must be defined, otherwise the system automatically assigns an index.

4. ADSL $2+$ configuration port interface: this step consists in configuring the ADSL 2+ port interface card and the slot in the MSAN (Table 9).

Table 9. Configuration of an ADSL 2+ port interface

\begin{tabular}{|l|l|}
\hline MSAN Smart AX MA5600T & MSAN C300M \\
\hline huawei(config)\#interface adsl & ZXAN(config)\#interface adsl_ \\
$0 / 4$ & $1 / 4 / 1-64$ \\
\hline
\end{tabular}

5. Adding VPI and VCI: Creation of FAI tunnel corresponding to our MSAN (Table10).

Table 10. Tunnel FAI with VPI and VCI ports

\begin{tabular}{|l|lllll|}
\hline MSAN Smart AX MA5600T & MSAN C300M & & \\
\hline multi-service-port vlan 103 & atm pve 1 vpi 0 vci 38 \\
board 1-18 vpi 0 vci 38 rx-cttr 6 & switchport default vlan 1115 pvc \\
tx-cttr 6 & 1 \\
\hline
\end{tabular}

Configuration of the VoIP voice service based on the SIP protocol: we have chosen as an example to configure the SIP protocol or we will try to summarize the main steps of configuration according to (Smart AX MA5600T and C300M) (Table 11) [7].
Table 11. VoIP based on the SIP protocol

\begin{tabular}{|c|c|}
\hline MSAN Smart AX MA5600T & MSAN C300M \\
\hline $\begin{array}{l}\text { huawei(config)\#voip } \\
\text { huawei(config-voip)\#ipaddress media } \\
10.50 .1 .10 \text { 10.50.1.1 } \\
\text { huawei(config-voip)\#ip } \\
\text { signaling 10.50.1.10 } \\
\text { huawei(config)\#ip route-static } \\
\text { 10.10.1.0 255.255.0.0 10.50.1.1 } \\
\text { huawei(config)\#interface sip 0 } \\
\text { Are you sure to add the SIP } \\
\text { interface?(y/n)[n]:y } \\
\text { huawei(config-if-sip-0)\#if-sip } \\
\text { attribute basic } \\
\text { media-ip 10.50.1.10 signal-ip } \\
\text { 10.50.1.10 signal-port } 5555 \text { transfer } \\
\text { udp primary-proxy-ip1 10.10.1.1 } \\
\text { primary proxy port 5555 secondary- } \\
\text { proxy-ip1 10.10.1.2 secondary- } \\
\text { proxy-port 5555 home domain } \\
\text { MA5600T/MA5603T/MA5608T.com } \\
\text { sip profile-index 0 }\end{array}$ & $\begin{array}{l}\text { ZXAN(config-msag)\#voip } \\
\text { interface add ctrl-ip } \\
10.63 .198 .227 \text { ctrl-mask } \\
255.255 .255 .0 \quad \text { media-ip } \\
10.63 .198 .227 \text { media-mask } \\
255.255 .255 .0 \text { mgid } 1 \\
\text { ZXAN(config)\#ip route } \\
10.61 .198 .0 \quad 255.255 .255 .0 \\
10.63 .198 .254 \\
\text { ZXAN(config-msag)\#sip } \\
\text { user add } 31 \text { begin-index } 1 \\
\text { num } 64 \quad+8659167050110 \\
\text { digit map name SIPDM } \\
\text { password } 123456 \\
\text { Username } 111670501 \text { begin- } \\
\text { no } 10 \text { digitlen } 2 \text { type } 3 \\
\text { ZXAN(config-msag)\#sip } \\
\text { proxy-server add } 1 \\
10.61 .198 .200 \\
\text { ZXAN(config-msag)\#sip } \\
\text { user-agent add } 11 \text { port } 5060 \\
\text { domname } 10.61 .198 .200\end{array}$ \\
\hline
\end{tabular}

In conclusion, as part of the increasingly urgent need for multimedia services, several operators around the world have begun to deploy NGN architectures that meet the needs of their customers. The gold study of this project allowed us to show the importance of the implementation of the MSAN Multi-Service Access Node through the presentation of the two solutions HUAWEI and ZTE as well as the services that offer them to meet the demand of subscribers with a better signal quality. We proposed as a perspective of this work, to integrate IPv6 and ensure the optimization of routing with genetic networks, and IPMSAN outdoor solution near customers (in order to minimize fiber costs as well as those for a twisted cable).

\section{References}

[1] J. Atzwanger, Multi-Service Access Node: Soft switches ITSF ROM (Telekom Austria, 2009).

[2] B. Handley, Fujitsu Scient. Technical J. 42(4), 432 (2006).

[3] D.C. Eddine, Presentation MSAN type ZTE (MSG 5200 \& C300M).

[4] DSM-2128 IP DSLAM and MSAN (Multi Service Access Node), Smart solutions for smart networks (2018).

[5] Huawei, MSAN MA5600T Basic Configuration, Issue 1-00 (2010).

[6] Huawei, SmartAX MA5600T/MA5603T/MA5608T Multiservice Access Module: Commissioning and Configuration Guide V800R015C10, Issue 01 (2014-10-30).

[7] Loïc. Debourdeau, Le Protocole SIP (Session Initiation Protocol), RFC2543.

[8] Sennouni. Hassane, Implementation de la solution Dual Homing pour la diversification des liens Uplinks du MSAN MA5600T au sein du reseaux metro Ip d'IAM, Ecole Nationale des Sciences AppliquéesTanger (2014).

[9] ZTE, ZXA10 C300M, Configuration Manual (CLI) (2012-10-20). 